\title{
An optimal schedule model of multi-energy hubs network integrating solar energy
}

\author{
V.V. Thang \\ Department of Electric Power System, Thainguyen University of Technology, Vietnam
}

\begin{abstract}
Article Info
Article history:

Received Feb 29, 2020

Revised Mar 25, 2021

Accepted May 4, 2021

\section{Keyword:}

Multi-energy system

Multi-energy hubs network

Optimization

Solar resource

Emission

ABSTRACT

Recently, multi-energy systems based on energy hub are introduced because of significant benefits in reducing energy and emission cost. This paper proposed an optimal schedule model of multi-energy hubs networks consisting of energy hubs, renewable sources, and energy storage which are connected by electrical and natural gas distribution networks. In the proposed mixedinteger nonlinear programming model, the objective is to minimize the operation, energy, and emission costs of energy hubs with both renewable sources and storage and energy distribution networks. The proposed schedule framework allows simultaneously selections of optimal operation structure of EHs together with the optimal operation parameters of energy distribution networks and therefore this model can maximize the profit of the entire largescale multi-energy hubs network. Besides, the operation parameters and energy loss of both electrical and natural gas distribution networks are considered in conjunction with optimal operation of energy hubs and thus guarantee the operation and optimization of the network in all operational scenarios. The IEEE 5-bus test system is utilized to demonstrate the applicability of the proposed model. The simulation results show the feasibility of the proposed model, and demonstrate that the energy hubs, renewable sources, and energy storage in the proposed structure significantly enhance the efficiency of the multi-energy hubs network by reducing not only energy and operation costs but also emission.
\end{abstract}

Copyright $(0) 2021$ Institute of Advanced Engineering and Science. All rights reserved.

\section{Corresponding Author:}

V.V. Thang,

Department of Electric power system,

Thainguyen University of Technology, Vietnam

Email: thangvvhtd@tnut.edu.vn

\section{INTRODUCTION}

Energy Hub (EH) has been introduced and applied in multi-energy systems in combination with renewable resources because of its significant benefits in reducing energy and emission cost [1]-[3]. Within EHs, multiple energy types can be converted, conditioned, and stored to optimize the use of energy resources, enhance efficiency, reduce emissions and costs, and increase the reliability of energy systems. Although efficiency is improved, the integration of EHs and distribution networks still brings a lot of challenges to system operation. Therefore, the operation and planning of systems containing different energy carriers have been studied and presented in many publications.

The principles to construct simulation models of components in an integrated multi-energy system based on the energy hub concept have been introduced in [4]. A multi-energy system should be structurally divided into several functional blocks concerning different channels of energy types. The input and output parameters of the blocks are consistent and interconnected. The concept of energy hubs, the research gaps remaining in the areas of modelling, optimization, and energy hub application are present in this research [5]. This research presents several assessment studies regarding the scheduling scheme of energy hubs. Controlling technologies were introduced but there is a lack of research on modelling, optimization, and energy hub application. Similarly, the characteristics of energy system models and existing modelling tools together with challenges in the field of modelling and optimization of the energy systems are also introduced in the studies [6][7]. There 
are six major challenges in the field of energy system modelling: closed models and modelling tools (i), data quality and transparency (ii), unreliable assumptions (iii), complexity (iv), conflicting interests (v), and the need for coding (vi).

Despite these challenges, several studies in energy system planning and operation have been carried out regarding the scheduling and control methodologies for energy hubs. However, there is a lack of tools for energy system modelling and optimization, which creates difficulties in the operation and planning of the energy hubs or energy systems. The structure and optimal operating schedule of residential EHs are introduced in [8] with input energy from electricity and natural gas to supply the electricity and heat loads. This study aims to optimize the total energy costs of EH on a typical day of the year that the parameters change under each hour in the day. To reduce the computational burden, nonlinear terms are replaced by the continuous variable and thus this model is reformulated as a MILP problem. Similarly, the research [9] proposes a residential EH structure in consideration to solar energy and cooling demand of consumers with a mathematical model to optimize the energy cost of EH. The storage systems are also integrated into multi-carrier EHs to improve the efficiency of the system. The objective is to minimize total operational cost with different constraints [10][11]. The management model for optimal scheduling of a multi-carrier energy hub proposed to optimal schedule and manage problem was modelled as an MINLP optimization problem. An optimized operation model is presented for combined cool, heat, and power systems in which renewable energy sources including photovoltaic (PV) and wind turbine (WT), and storages are added and presented in [12]. The objective of optimal operation of an $\mathrm{EH}$ is to minimize daily operation costs (costs of electricity, gas, and carbon emission). Several energy hubs with different constructions, ratings, parameters, and constraints are simulated through MILP to show the efficiency of this model and how it can be generalized to larger and more complex systems.

Besides, the uncertainty of parameters displayed by stochastic models is integrated into the stochastic optimization framework of EH [13]. The objective function is to minimize the operation cost of EH, which consists of imported energy carriers costs in addition to devices and operation costs. The authors in research [14] introduced an optimal operation model of multi-carrier energy systems in the presence of wind farms, electrical and thermal storage systems, electrical and thermal demand response programs, electricity market, and thermal energy market. The stochastic parameters of energy hubs are integrated into the optimal problem and thus force all variables to be scenario-based. The objective function of this optimization problem will consist of imported energy carriers cost in addition to devices and programs operation cost. The structure of an EH with renewable energy sources such as PV and WT is integrated into the proposed model. It also introduces the economic objective function and constraints in [15][16]. Model is provided for scheduling of EH considering economic and environmental constraints in the presence of market price uncertainty and multidemand response programs.

In addition, the optimization problem based on modelling uncertainty parameters is proposed to maximize the profit of the energy hub [17]. The model for the optimization problem is MILP. The simulation results of the proposed model show an increase in energy hub profit, reducing the cost of power purchased from the electricity grid as well as decreasing the cost of operation. Moreover, a novel technology which is power-togas storage is utilized in energy hub structure to cover increasing renewable energy resource variability in power system operations [18]. A stochastic approach is applied to handle the uncertain parameters of EH in which the objective function of the proposed problem is to minimize the total operation cost of EH, which is modelled as a MILP problem model. The calculation results showed the effectiveness of the load shifting based on the demand response program and the P2G storage system used as a new resource that makes a connection between electrical and natural gas networks to reduce the operation cost of the EH. Previous studies indicated the importance and effectiveness of the optimal scheduling of EH as one of the key infrastructures that enable the interconnection between different energy types in a multi-energy system. However, the development of a model that enables to optimally schedule for multi-energy hubs network remains a critical issue for operators.

In large areas, energy networks must supply a lot of loads with a large distance between them. Therefore, many EHs are connected to large-scale multi-energy hubs network by distribution networks to supply energy to customers. A decomposed solution to optimally operate the large-scale energy hubs network is proposed in research [19][20] with the minimum objective function of energy cost. The optimization framework enables coupled power flow studies on different energy infrastructures such as electric and natural gas. This is a largescale nonlinear problem. Thus, the decomposed solution is utilized through a robust optimization technique, which is the multi-agent genetic algorithm to reduce the computational burden while major advantages of simultaneous analysis of multi-carrier energy systems would not be sacrificed. However, optimal power flow of multiple-energy carrier networks is only considered after the EHs operation optimized in the previous stage and thus the results can cause a large error. Similarly, in [21] and [22], the two-stage optimal scheduling model for a multi-energy hub system network to improve the economic benefits of the EH and the distribution network is presented with a demand response strategy. The first stage is to schedule for each EH through input initial energy prices, initial energy loads, distribution network parameters, and parameters of the EH. The second 
stage is to schedule for the exchange power between the distribution network and the EHs with the constrained condition of each EH obtained in the first stage. In these studies, the energy storage devices, which have a significant influence on the effectiveness of the EH integrated renewable sources, were not considered.

The loss cost of networks and renewable sources is simultaneously considered in an optimal problem which finds the minimum of energy and emission costs [23]. The game theory was used to solve the optimal scheduling of a multi-energy hub system in this study. The results showed the optimal scheduling of multienergy hub systems using game theory has economic value and certain practical engineering value. A mathematical model for the optimal energy management of an urban energy network with energy hubs has also been presented in [24]. The objective function which seeks to minimize the total operational and maintenance costs of the network of energy hubs is integrated into MILP and solved in the General Algebraic Modeling Software (GAMS). The authors in the study [25] developed the model and optimization of a complex network of energy hubs while considering the integration of both electrical and natural gas systems. The optimal model includes an objective function to minimize both cost terms and terms related to emissions and constraints. The function is applied to compute two cases using actual data. Results showed the creation of a network of energy hubs is an effective strategy for reducing system total costs and emissions. Moreover, using a complex network of energy hubs has qualitative benefits of increasing reliability and reducing electrical grid congestion. The change of loads and output power of sources is analysed under typical curves of the day. However, the energy loss is only determined under loss factors of electricity and natural gas flow into the EHs, feeders, and pipelines whereas they ignored the operation parameters of energy networks such as node voltages and optimal power flow of the electrical network, pressures of pipelines in natural gas network.

A novel technique for optimum operation and configuration of multiple energy hubs is also proposed with different types of energy sources, generation, and multi-type energy storage devices to feed electrical, heating, and gas demands [26]. The optimal framework is a multi-objective optimization problem formulated to maximize social welfare and minimize emissions. The uncertainties of renewable energy sources were considered in the proposed model and the genetic algorithm is used to solve the overall optimization problem. The Newton-Raphson method was used to solve the problem of heat and gas flow to determine the state variables and check the constraints of the system. The different configurations of the EH are assumed and then they are comparatively analysed by the objective function which maximizes social welfare and minimizes emissions. The results proved that increasing the system size improved the performance parameters and $\mathrm{EH}$ stability. However, only five assumed structures are used to test the effects of equipment in EH of energy systems, and therefore optimizing the structure of the EH in the optimal problems of multi-energy hub system networks is not completed.

In general, the above studies have shown the effectiveness of the optimal operation methods for each $\mathrm{EH}$ individually as well as multiple EHs connected to large-scale networks. However, in proposed optimal operation models for large-scale multi-energy hubs networks, most of them only individually optimize the EHs or distribution networks in two-stage models without optimizing the cost of the entire system. Moreover, the operation parameters of energy networks are not considered thus the operation cannot be guaranteed in practice. In this study, the structure and optimal schedule model of a multi-energy hubs network consisting of EHs, renewable sources, energy storages, and both electrical and natural gas networks are proposed. The main contributions of the research are as follows:

- Optimizing the energy and operation costs of all networks including EHs. Renewable sources and storage and energy distribution networks consisting of electric and natural gas distribution networks are simultaneously determined in a mixed-integer nonlinear programming problem.

- The optimal operation structure of the EHs is selected with the optimal operation parameters of energy distribution networks and therefore this model can maximize the profit of the entire large-scale multienergy hubs network.

- The operation parameters and energy loss of both electrical and natural gas distribution networks are simultaneously considered with EHs and thus guarantee the operation and optimization of the network in all operational scenarios.

The remainder of this paper is organized as follows. In Section 2, the network and energy hub modelling formulation is presented and the mathematical formulation of this optimal schedule problem is established in Section 3. Section 4 presents a case study with numerical results. Finally, the conclusions are presented in Section 5 .

\section{NETWORK AND ENERGY HUB MODELING FORMULATION}

In general, the structure of an energy hub network often includes the different EHs which are connected through different transmission networks such as electrical distribution network (EDN) and gas 
distribution network (GDN). To optimally schedule the energy hub network, the mathematical modelling of the network and the EHs is described.

\subsection{Energy hub modeling}

Energy hub is introduced to describe the interconnection between consumers, producers, converter devices, storage devices, and transmission devices in different ways as figure 1 [1]. Within each EH, the input energy forms are converted, conditioned, and stored to meet the various forms of output demand. To model the transformations that occur within the energy conversion of an $\mathrm{EH}$, the coupling matrix is utilized to describe the relationship between the input carriers and the output demand as shown in equation (1). Where, the entries $C_{i j}$ relate the $i^{\text {th }}$ input power $\left(P_{i}\right)$ to the $j^{\text {th }}$ output power $\left(P_{L j}\right)$.

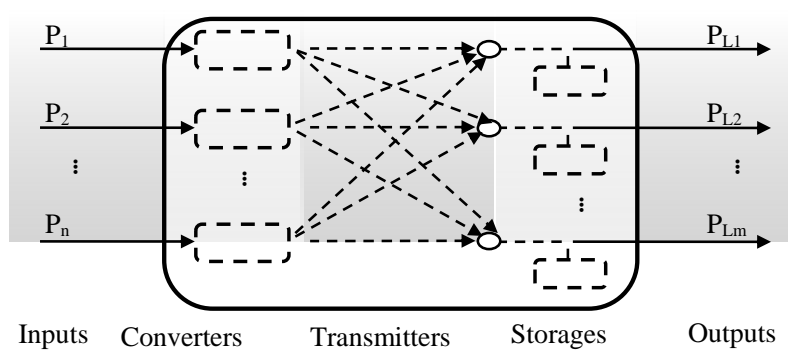

Figure 1. General structure of an $\mathrm{EH}$

To effectively improve the EHs, the different multi-energy forms are incorporated within the residential, commercial, agricultural, and industrial sectors. A typical $\mathrm{EH}$, as shown in figure 2, widely has been applied in recent researches [9][28]. In this structure, the input energy forms include the electricity and nature gas together with solar energy source are converted and conditioned by combined heat and power (CHP), gas boiler (GB), Electrical Chiller (EC), Absorption Chiller (AC), a solar heat exchanger (SHE), transformer (TR) and stored/generated by BESS to supply for loads consisting of electricity, heating, and cooling.

$$
\begin{gathered}
{\left[\begin{array}{cccc}
C_{11} & C_{12} & \cdots & C_{1 m} \\
C_{21} & C_{22} & \cdots & C_{2 m} \\
\vdots & \vdots & \vdots & \vdots \\
C_{n 1} & C_{n 2} & \cdots & C_{n m}
\end{array}\right]\left[\begin{array}{c}
P_{1} \\
P_{2} \\
\vdots \\
P_{m}
\end{array}\right]=\left[\begin{array}{c}
P_{L 1} \\
P_{L 2} \\
\vdots \\
P_{L n}
\end{array}\right]} \\
\eta_{e c}=\frac{C O P_{e c}}{1+C O P_{e c}} ; \eta_{a c}=\frac{C O P_{a c}}{1+C O P_{a c}}
\end{gathered}
$$

Based on the structure of $\mathrm{EH}$ in figure 2 and the coupling matrix in equation (1), the energy balance constraints of $\mathrm{EH} i$ are proposed as the following expression:

$$
\left\{\begin{array}{l}
P_{i, h}^{\mathrm{le}}=\left(1-v_{i, h}^{e c}\right) \cdot\left(\eta_{\mathrm{tr}} \cdot P_{i, h}^{\mathrm{e}}+v_{i, h}^{g} \cdot P_{i, h}^{\mathrm{g}} \cdot \eta_{c h p}^{\mathrm{ge}}+P_{i, h}^{\mathrm{pv}}\right)+P_{\mathrm{ch} . i, h}^{\mathrm{be}} \cdot \gamma_{\mathrm{ch} . \mathrm{i}, \mathrm{h}}^{\mathrm{be}}-\eta_{\mathrm{be}} \cdot P_{d i s . i, h}^{\mathrm{be}} \cdot \gamma_{\mathrm{dis} . \mathrm{i}, \mathrm{h}}^{\mathrm{be}} \\
P_{i, h}^{\mathrm{lh}}=\left[\left(v_{i, h}^{g} \cdot \eta_{\mathrm{chp}}^{\mathrm{gh}}+\left(1-v_{i, h}^{g}\right) \cdot \eta_{\mathrm{gb}}\right) \cdot P_{i, h}^{\mathrm{g}}+P_{i, h}^{\mathrm{she}}\right] \cdot\left(1-v_{i, h}^{a c}\right) \\
P_{i, h}^{\mathrm{lc}}=v_{i, h}^{e c} \cdot\left(\eta_{\mathrm{tr}} \cdot P_{i, h}^{\mathrm{e}}+v_{i, h}^{g} \cdot P_{i, h}^{\mathrm{g}} \cdot \eta_{c h p}^{\mathrm{ge}}+P_{i, h}^{\mathrm{pv}}\right) \cdot \eta_{\mathrm{ec}}+\left[\left(v_{i, h}^{g} \cdot \eta_{\mathrm{chp}}^{\mathrm{gh}}+\left(1-v_{i, h}^{g}\right) \cdot \eta_{\mathrm{gb}}\right) \cdot P_{i, h}^{\mathrm{g}}+P_{i, h}^{\mathrm{she}}\right] \cdot v_{i, h}^{a c} \cdot \eta_{\mathrm{ac}}
\end{array}\right.
$$

$\forall i \in N, h \in H$

Where, $P_{i, h}^{e}$ and $P_{i, h}^{g}$ are the amount of electricity and natural gas of EH $i$ purchased from the market in hour $h$, respectively. Similarly, $P_{\mathrm{ch} . i, h}^{\mathrm{be}}$ and $P_{\mathrm{dis} . i, h}^{\mathrm{be}}$ are the charge/discharge power of BESS in EH $i$, hour $h$, respectively. $v_{i, h}^{a c}, v_{i, h}^{e c}$ and $v_{i, h}^{g}$ are the dispatch ratios of electricity, heat and natural gas at hour $h$ of AC, EC and CHP, respectively. $\eta_{\text {chp }}^{\text {ge }}$ and $\eta_{\text {chp }}^{\text {gh }}$ are conversion efficiency from the gas to electricity and heat of the CHP, respectively. $\eta_{t r}, \eta_{g b}$ and $\eta_{b e}$ are efficiency of transformers, GB and BESS, respectively. $N$ is total EHs of network and $H$ is total number of hours a day (24 hours). $\eta_{e c}$ and $\eta_{a c}$ are efficiency of EC and AC which usually are calculated based on coefficient of performance (COP) as equation follows (2). Where, $C O P_{e c}$ and $C O P_{a c}$ are the coefficient of performance for $\mathrm{EC}$ and $\mathrm{AC}$, respectively. 


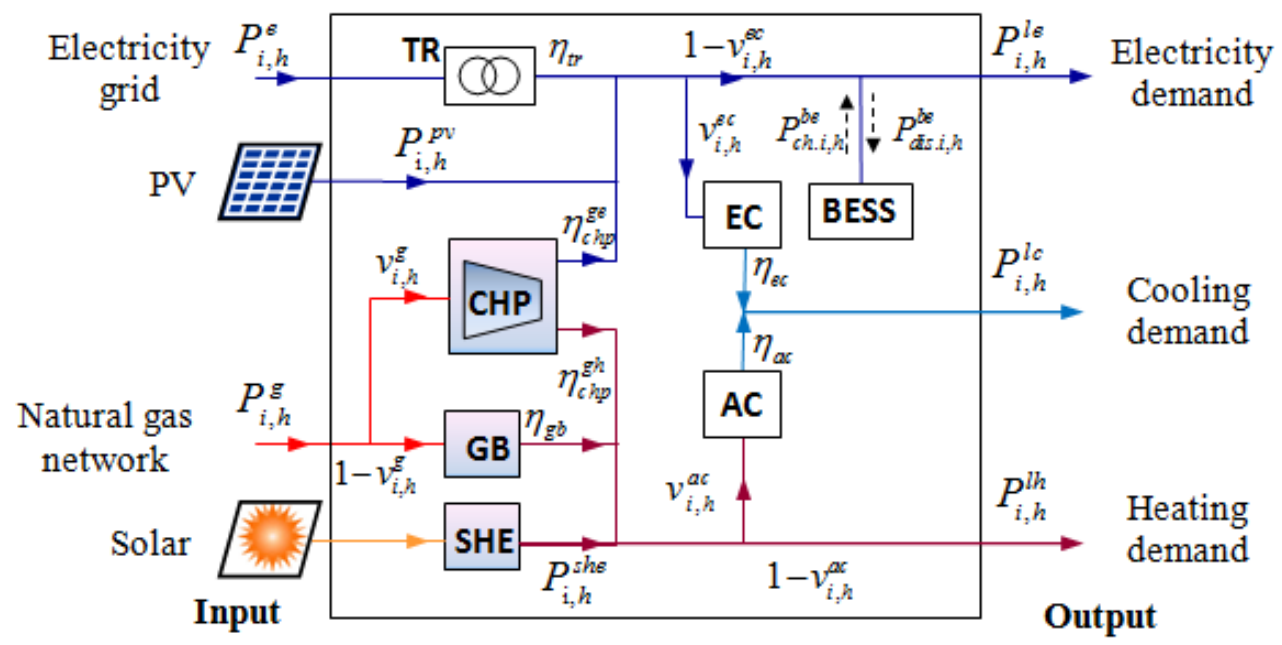

Figure 2. Structure of typical EH

The final, the power of electrical, heating and cooling loads at time $h$ are denoted by $P_{i, h}^{l e}, P_{i, h}^{l h}$, and $P_{i, h}^{l c}$, respectively. They are expressed through factors that change under hour in day and are denoted by $k_{h}^{l e}$ (for electrical load), $k_{h}^{l h}$ (for heating load) and $k_{h}^{l c}$ (for cooling load) and maximum value of loads $\left(P_{\text {max. } i}^{l e}, P_{\text {max.i }}^{l h}, P_{\operatorname{max.i}}^{l c}\right)$ as represent in equation (4).

$$
\begin{aligned}
& P_{i, h}^{l e}=k_{h}^{l e} \cdot P_{\text {max. } i}^{l e} ; P_{i, h}^{l h}=k_{h}^{l h} \cdot P_{\max . i}^{l h} ; P_{i, h}^{l c}=k_{h}^{l c} \cdot P_{\max . i}^{l c} \\
& \forall i \in N, h \in H
\end{aligned}
$$

The BESS can charge when the high output power of PV or low loads and electrical price. On the contrary, when the peak loads with high electrical price, or low output power of PV, the BESS discharge to reduce the cost of the system. The charge/discharge decision of BESS is expressed through two binary variables $\left(\alpha_{c h . h}, \alpha_{\text {dis.h }}\right)$ and the balance between charge and discharge energy in the calculation cycle represented in equations (5) and (6) [29]. Where, $P_{c h . i, h}^{b e}$ and $P_{d i s ., h}^{b e}$ are the charge/discharge power at hour $h$ and are limited by maximum power of $\operatorname{BESS}\left(P_{\max . i}^{b e}\right)$.

$$
\begin{aligned}
& \sum_{h=1}^{H} \alpha_{c h . i, h} . P_{c h . i, h}^{b e} \cdot \eta_{b e}=\sum_{h=1}^{H} \alpha_{\text {dis. } ., h} . P_{d i s . i, h}^{b e} ; \quad \alpha_{d i s . h}+\alpha_{c h . h} \leq 1 \\
& \forall i \in N, h \in H \\
& 0 \leq P_{c h . i, h}^{b e} \leq P_{\text {max. } i .}^{b e} ; 0 \leq P_{d i s . i, h}^{b e} \leq P_{\max . i}^{b e} \quad \forall i \in N, h \in H
\end{aligned}
$$

The stored energy level of BESS is limited by the SOC value which is increased in the charge time and decreased in the discharge time as represented in the equation (7) [30]. Besides, to ensure the lifetime of BESS, the stored energy has the following lower and upper bounds as equation (8). In which, the minimum and maximum SOC coefficient is $k_{s o c}^{\min }$ and $k_{s o c}^{\max }$, respectively. $E_{i, h}^{b e}$ and $E_{\max , i}^{b e}$ are stored energy at hour $h$ and the maximum capacity of BESS in EH $i$.

$$
\begin{aligned}
& E_{i, h}^{b e}=E_{i, h-1}^{b e}+\alpha_{c h . h} \cdot P_{c h . i, h}^{b e} \cdot \eta_{b e}-\alpha_{d i s . h} \cdot P_{d i s . i, h}^{b e} \quad \forall i \in N, h \in H \\
& k_{s o c}^{\min } \cdot E_{\max . i}^{b e} \leq E_{i, h}^{b e} \leq k_{s o c}^{\max } \cdot E_{\max . i}^{b e} ; \quad E_{i, H}^{b e}=E_{i, 0}^{b e} \quad \forall i \in N, h \in H
\end{aligned}
$$

\subsection{Solar energy resource modeling}

The solar irradiance is uncertain and always varies with an hour in day and season in the year. While the output power of PV depends on solar irradiance and the operating ambient temperature and thus it also varies with an hour in day and season in the year. Therefore, the output power of PV in each hour $\left(P_{h}^{p v}\right)$ is analysed as expression (9) [31][32] with power temperature coefficient $\left(\alpha_{\theta}\right)$ and standard ambient temperature 
$\left(\theta_{a m}\right)$. In which, $P_{r}^{p v}$ and $I_{r}$ are rated power and solar irradiance of PV. $I_{h}$ and $\theta_{h}$ are operation irradiance and temperature at hour $h$.

$$
P_{h}^{p v}(I, \theta)=P_{r}^{p v} \cdot \frac{I_{h}}{I_{r}}\left[1+\alpha_{\theta}\left(\theta_{h}-\theta_{a m}\right)\right]
$$

Similarly, the thermal power generated by SHE also varies with the solar irradiance [24] as well as operating temperature because the collector efficiency is a linear function of operation temperature [20]. Therefore, the output power of SHE can be described by functions of time as equation (10). Where, $\theta_{s t}$ is the stagnation temperature and $P_{r}^{\text {she }}$ is the rated output power of the SHE corresponding to standard solar irradiance $I_{r}$.

$$
P_{h}^{s h e}(I, \theta)=P_{r}^{s h e} \cdot \frac{I_{h}}{I_{r}}\left[1-\left(\frac{\theta_{h}-\theta_{a m}}{\theta_{s t}-\theta_{a m}}\right)\right]
$$

\subsection{Modeling of distribution network among energy hubs}

The EH network composes of interconnected multi-energy hubs by the electrical and gas distribution networks. In each node, the EH is connected with both electrical and gas distribution networks. The input power of EHs receives from the EDN is the electrical power $\left(P_{i, h}^{e}\right)$ and GDN is the gas power $\left(P_{i, h}^{g}\right)$. Hence, $P_{i, h}^{e}$ and $P_{i, h}^{g}$ become loads of EDN and GDN, respectively.

\subsubsection{Modeling of electricity network}

The AC nonlinear power flow model of EDN with $N$ nodes is presented in equation (11) [34]. In which, $P_{i, h}^{S}$ and $Q_{i, h}^{S}$ are active and reactive power that the EDN is received from the utility grid through connection node. $U_{i, h}, \delta_{i, h}$ are module and angle of bus voltage. $Y_{i j}, \theta_{i j}$ are the magnitude and angle of the admittance matrix element formulated by the feeder impedances. The model improves the accuracy results because the effects of both active and reactive power to losses are reduced.

$$
\begin{aligned}
& P_{i, h}^{S}-P_{i, h}^{e}=\sum_{j=1}^{N}\left|Y_{i j}\right| \cdot\left|U_{i, h}\right| \cdot\left|U_{j, h}\right| \cdot \cos \left(\theta_{i j}-\delta_{j, h}-\delta_{i, h}\right) \\
& Q_{i, h}^{S}-Q_{i, h}^{e}=-\sum_{j=1}^{N}\left|Y_{i j}\right| \cdot\left|U_{i, h}\right| \cdot\left|U_{j, h}\right| \cdot \sin \left(\theta_{i j}-\delta_{j, h}-\delta_{i, h}\right)
\end{aligned}
$$

To ensure the devices of EDN are not overloaded by thermal limit, the operation power flow in feeders $\left(S_{i j, h}^{f}\right)$ and connectable substation $\left(S_{i, h}^{S}\right)$ are limited as shown in equation (12). Where, $S_{i j \text { max }}^{f}$ and $S_{i \cdot \max }^{S}$ are the limited capacity of feeders and connectable substation.

$$
S_{i j, h}^{f} \leq S_{i j \text { max }}^{f} ; \quad S_{i, h}^{S} \leq S_{i . \max }^{S} \quad \forall i j \in N, h \in H
$$

Additionally, the voltage profile at buses must be guaranteed in a limit allowing the ordinary operation of the EDN as presented in equation (13). The voltage at the substation bus connected to utility grid is assumed to constant while the voltage at load buses often varies under the change of load. Therefore, these voltages are limited between minimum voltage profile $U_{\min }$ and maximum voltage profile $U_{\max }$.

$$
\begin{aligned}
& \left|U_{i, h}\right|=\text { constant } \quad \forall i \in N_{S}, h \in H \\
& U_{\text {min }} \leq\left|U_{i, h}\right| \leq U_{\max } \quad \forall i \in N, h \in H
\end{aligned}
$$

\subsubsection{Modeling of natural gas network}

A typical GDN consists of gas producers, compressor stations, pipelines, and customers or loads. The gas flow in the pipelines to connected nodes can be expressed as equation (14) [33][34]. Where, $c_{k}$ is the pipeline constant and $k_{i j}^{p l}$ is the property of the fluid. $p_{i, h}$ and $p_{j, h}$ are pressures at node $i$ and $j$ in hour $h$, respectively.

$$
\begin{aligned}
& P_{i j, h}^{p l}=k_{i j}^{p l} \cdot \mathrm{c}_{k} \sqrt{\alpha_{i j}\left(\mathrm{p}_{i, h}^{2}-\mathrm{p}_{j, h}^{2}\right)} ; \quad \alpha_{i j}= \begin{cases}+1 & \text { if } \quad p_{i, h} \geq p_{j, h} \\
-1 & \text { else }\end{cases} \\
& \forall i j \in N, h \in H
\end{aligned}
$$


Due to decreased gas pressure during transmission, the compressor can be utilized to ensure sufficient gas pressure. Hence, the compressor demand can be approximated as equation (15) with the constant of a compressor, $k_{c o}$.

$$
P_{i j, h}^{p l . c o}=k_{c o} \cdot P_{i j, h}^{p l} \cdot\left(p_{j, h}-p_{i, h}\right) \quad \forall i j \in N, h \in H
$$
constraints:

Besides, the pressure at each node and gas flow must satisfy the limit of pipelines as the following

$$
\begin{aligned}
& p_{\min . i} \leq p_{i, h} \leq p_{\max . i} ; \quad P_{\min . i j}^{p l} \leq P_{i j, h}^{p l} \leq P_{\max . i j}^{p l} \\
& \forall i j \in N, h \in H
\end{aligned}
$$

Where, $p_{\min . i}$ and $p_{\max . i}$ are minimum and maximum pressure of the pipeline. $P_{\min . i j}^{p l}$ and $P_{\max . i j}^{p l}$ are minimum and maximum power of the gas flow in pipeline $i j$.

\section{MATHEMATICAL MODEL}

Due to different energy carriers available at the inputs as well as outputs and the possibility of internal power conversion of the EHs, the energy hub network has high flexibility and thus the power flows in the network can be controlled within a certain degree of freedom to optimize the operation cost as well as technic parameters of the system. Hence, the problem of optimal operation of the EHs and energy networks is modelled as a MINLP problem in this section.

\subsection{Objective function}

The objective function to be minimized takes into account cost terms of energy and operation, and terms related to emissions in a computed cycle ( 24 hours) as follows:

$$
M i n F_{E H N}=\sum_{h=1}^{H}\left(F_{e n . h}^{e g}+F_{o p . h}^{s e}+F_{e m . h}\right)
$$

Where, $F_{e n . h}^{e g}$ is electrical and natural gas energy cost purchased from the market in each hour and it is computed as equation (18) with electrical price $\lambda_{h}^{e}$ and gas price $\lambda_{h}^{g}$.

$$
\begin{aligned}
& F_{e n . h}^{e g}=\lambda_{h}^{e} \sum_{j=1}^{N_{s}} P_{j, h}^{s e}+\lambda_{h}^{g} \sum_{j=1}^{N_{s}} P_{j, h}^{\mathrm{sg}} \\
& \sum_{j=1}^{N_{s}} P_{j, h}^{s e}=\sum_{i=1}^{N_{E H}} P_{i, h}^{e}+\sum_{i=1}^{N} \sum_{j=1}^{N} \Delta P_{i j, h}^{e} \\
& \sum_{j=1}^{N_{s}} P_{j, h}^{\mathrm{sg}}=\sum_{i=1}^{N_{E H}} P_{i, h}^{e}+k_{\Delta}^{g} \sum_{i=1}^{N} \sum_{j=1}^{N} P_{i j, h}^{p l}
\end{aligned}
$$

The electrical price is often the Time-Of-Use (TOU) and is the simplest form of the dynamic price while natural gas prices are constant [24][25][34]. $\Delta P_{i j, h}^{e}$ is the power loss of EDN is determined by the nonlinear power flow model in (11) while the loss in GDN is based on loss coefficient $k_{\Delta}^{g}$ [23].

$F_{o p . h}^{s e}$ is the operation cost of solar energy systems (PV and SHE) in hour $h$ defined as equation (19) and $F_{e m . h}$ is emission cost in hour $h$ of the energy hub network expressed as (20). In which, $C_{0}^{p v}$ and $C_{0}^{\text {she }}$ are operation cost factors based on the output power of the PV and SHE, respectively. $\xi_{s e}$ is the emission coefficient of solar energy. $\xi_{e}$ and $\xi_{g}$ are the emission coefficient of electrical and natural gas energy, respectively. $\rho_{e m}$ is the emission tax probably enforced by the government.

$$
\begin{aligned}
& F_{o p . h}^{s e}=\sum_{i=1}^{N}\left(C_{0}^{p v} \cdot P_{i, h}^{p v}+C_{0}^{s h e} \cdot P_{i, h}^{\text {she }}\right) \\
& F_{e m . h}^{e g}=\sum_{i=1}^{N} \rho_{e m}\left[\xi_{s e} \cdot\left(P_{i, h}^{p v}+P_{i, h}^{s h e}\right)+\xi_{g} \cdot P_{i, h}^{g}+\xi_{e} \cdot P_{i, h}^{e}\right]
\end{aligned}
$$




\subsection{Constraints}

According to the network and EHs modelling explained in Section 2, the electrical and thermal balance constraints as well as the operational constraints for each EH are presented in equations (3)-(8). Similarly, the EDN constraints include equations (11)-(13) and the GDN constraints consist of equations (14)-(16).

Additionally, the supply capacity of the systems is limited by the capacity and energy stored as expression (21) with the maximum allowable power in node $i$ of electricity ( $\left.S_{\text {max.i }}^{e}\right)$ and natural gas $\left(P_{\text {max.i }}^{g}\right)$.

$$
\begin{aligned}
& S_{i, h}^{e} \leq S_{\text {max.i }}^{e} ; \quad P_{i, h}^{g} \leq P_{\text {max.i }}^{g} \\
& \forall i \in N_{S}, h \in H
\end{aligned}
$$

Similarly, the power limit of the CHP and GB is presented in constraints (22) with the maximum power of the CHP ( $\left.P_{\max . \mathrm{i}}^{c h p}\right)$ and GB $\left(P_{\max . \mathrm{i}}^{g b}\right)$. The power limit of the EC and AC is limited by maximum power $\left(P_{\max . \mathrm{i}}^{e c}\right)$ and $\left(P_{\max . \mathrm{i}}^{a c}\right)$, respectively.

$$
\begin{aligned}
& P_{i, h}^{g} \cdot v_{i, h}^{g} \leq P_{\max . i}^{c h p} ; P_{i, h}^{g} \cdot\left(1-v_{i, h}^{g}\right) \leq P_{\max . i}^{g b} \\
& v_{i, h}^{e c} \cdot\left(\eta_{\mathrm{tr}} \cdot P_{i, h}^{\mathrm{e}}+v_{i, h}^{g} \cdot P_{i, h}^{\mathrm{g}} \cdot \eta_{c h p}^{\mathrm{ge}}+P_{i, h}^{\mathrm{pv}}\right) \leq P_{\max . i}^{e c} \\
& {\left[\left(v_{i, h}^{g} \cdot \eta_{\mathrm{chp}}^{\mathrm{gh}}+\left(1-v_{i, h}^{g}\right) \cdot \eta_{\mathrm{gb}}\right) \cdot P_{i, h}^{\mathrm{g}}+P_{i, h}^{\mathrm{she}}\right] \cdot v_{i, h}^{a c} \leq P_{\max . i}^{a c}} \\
& \forall i \in N, h \in H
\end{aligned}
$$

The optimal operation problem of the EHs is based on the ability to control the power flow of conversion devices. Therefore, the conversion limits of the devices at each hour are expressed as:

$$
0 \leq v_{i, h}^{g} \leq 1 ; \quad 0 \leq v_{i, h}^{e c} \leq 1 ; \quad 0 \leq v_{i, h}^{a c} \leq 1
$$

\subsection{Solution method}

The proposed model is formulated with a nonlinear model mixed to integer variables to optimize the operation of each EH including energy conversion technologies as well as the energy hub network. The proposed model is formulated with a nonlinear model mixed to integer variables to optimize the operation of each EH including energy conversion technologies as well as the energy hub network. To solve the MINLP problem, a lot of solvers are presented in the existing literature [35]. In which, BONMIN is the best solver for solving the MINLP non-convex problems of GAMS environment that is a high-level modelling system for mathematical programming and optimization [36][37]. BONMIN implements three different algorithms to solve MINLPs consisting of simple branch-and-bound algorithms, outer-approximation-based decomposition algorithm, and outer-approximation-based branch-and-cut algorithm. It is not an exact solver only for convex problems but taking into consideration the values of the heuristic solutions to solve the problem efficiently for convergence compared to the other mention solvers or meta-heuristic algorithms. Additionally, the problems are successfully solved with the least computational burden [38]. For the above reasons, this research is directed towards the use of BONMIN solver to find out an optimal solution to the proposed problem.

\section{RESULTS AND DISCUSSION}

\subsection{Test structure and assumptions of case study}

To investigate the feasibility and efficiency of the proposed model, the IEEE 5-bus network structure is utilized in this research shown in figure 3 [39][40]. The parameters are changed and the GDN also is added to match the problem. In each node of the energy distribution network, an EH with a typical structure introduced in figure 2 is connected to receive the energy from a distribution network. The total electricity and natural gas of the energy hub network are purchased from the utility electrical grid and gas producer through electrical and gas substation, respectively. 


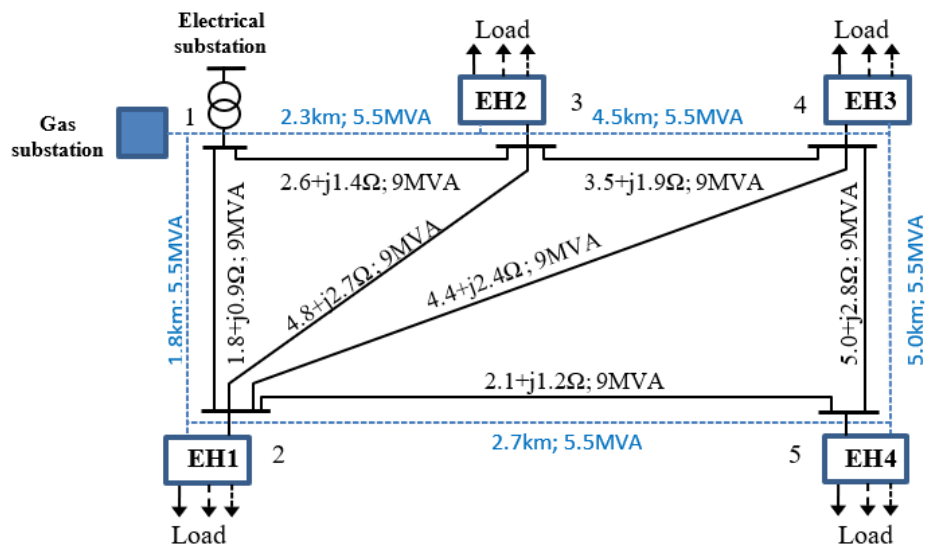

Figure 3. Typical multi-energy hubs network

The input parameters of EHs are shown in Table 1 [23]-[25]. In which, the total conversion efficiency of CHP is 0.85 with $40 \%$ converted to electricity and $45 \%$ converted to heat. The efficiency of EC and AC depends on the coefficient of performance (COP) of them and equal about $60 \%$. Similarly, the capacity limits of the equipment as well as the supply substations to ensure the proper operation of the EHs.

Input parameters of the distribution networks are assumed in figure 3, the normal voltage of the EDN is $22 \mathrm{kV}$ connected to the utility grid through substation at node 1 . Similarly, the gas substation at node 1 supplies for EHs through the pipelines of the GDN with a loss factor of about 5\% [23][25].

The energy demand of the EHs is assumed the difference and daily variation as shown in figure 4 with a power factor about 0.9. Similarly, the typical one-day variation of the PV, and SHE power is displayed in figure 5 and the daily energy price is presented in figure 6 . The natural gas prices are constant while the electrical prices are TOU prices with reactive power prices depending on average compensation for reactive power support which is calculated based on a support factor of about $10 \%$ of the active power prices [41][42].

Table 1. Parameters of the components in EHs

\begin{tabular}{llllllll}
\hline Parameter & Value & Parameter & Value & Parameter & Value & Parameter & Value \\
\hline$\eta_{t r}$ & 0.95 & $\eta_{a c}$ & 0.6 & $P_{\max }^{g}(M W)$ & 15 & $P_{b e}^{m a x}(M W)$ & 0.15 \\
$\eta_{e c}$ & 0.6 & $\eta_{b e}$ & 0.9 & $P_{e c}^{\max }(M W)$ & 2.5 & $E_{b e}^{m a x}(M W h)$ & 0.5 \\
$\eta_{c h p}^{g e}$ & 0.4 & $E_{\max }^{e}(M W h)$ & 300 & $P_{a c}^{m a x}(M W)$ & 2.5 & $P_{c h p}^{m a x}(M W)$ & 5 \\
$\eta_{c h p}^{g h}$ & 0.45 & $E_{\max }^{g}(M W h)$ & 250 & $P_{g b}^{\max }(M W)$ & 5 & $S_{t r}^{\max }(M V A)$ & 20 \\
$\eta_{g b}$ & 0.9 & $P_{\max }^{e}(M W)$ & 15 & & & & \\
\hline
\end{tabular}
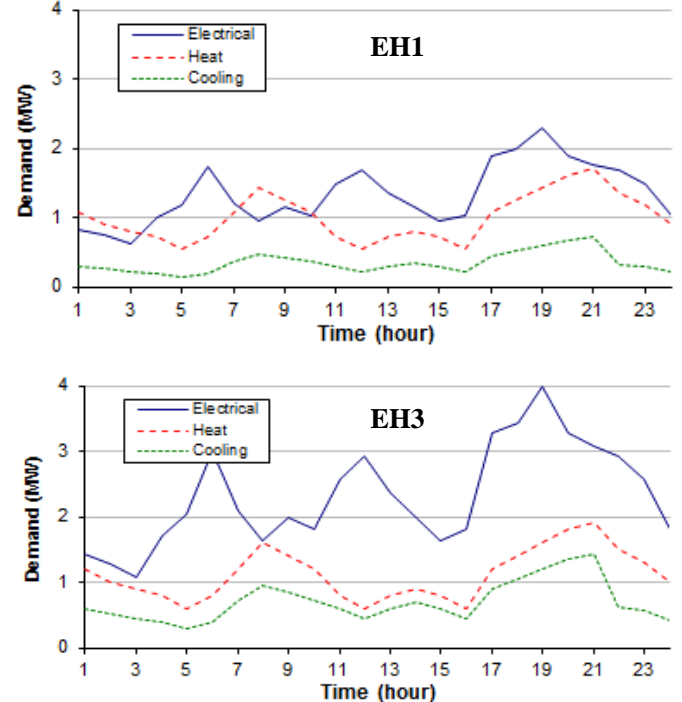
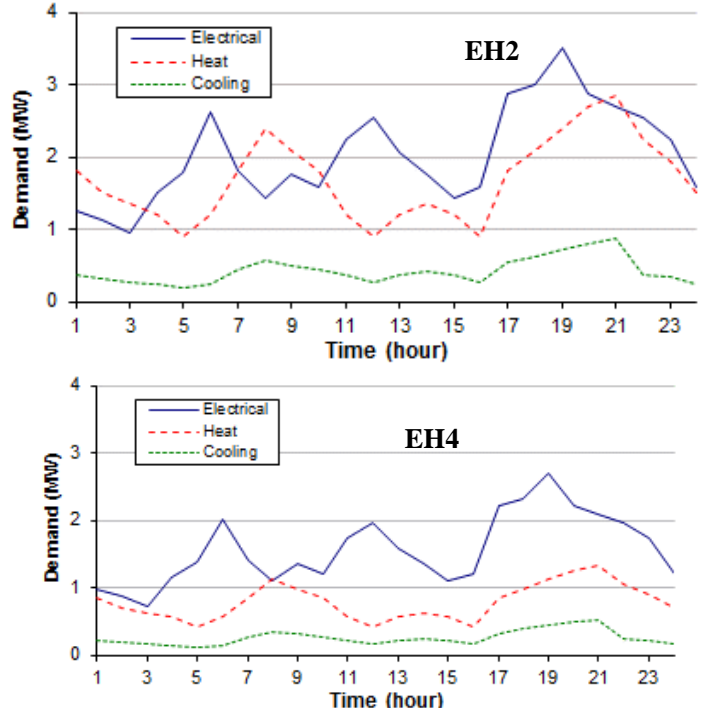

IJEEI, Vol. 9, No. 2, June 2021: 276-288 
Figure 4. Energy demand of EHs

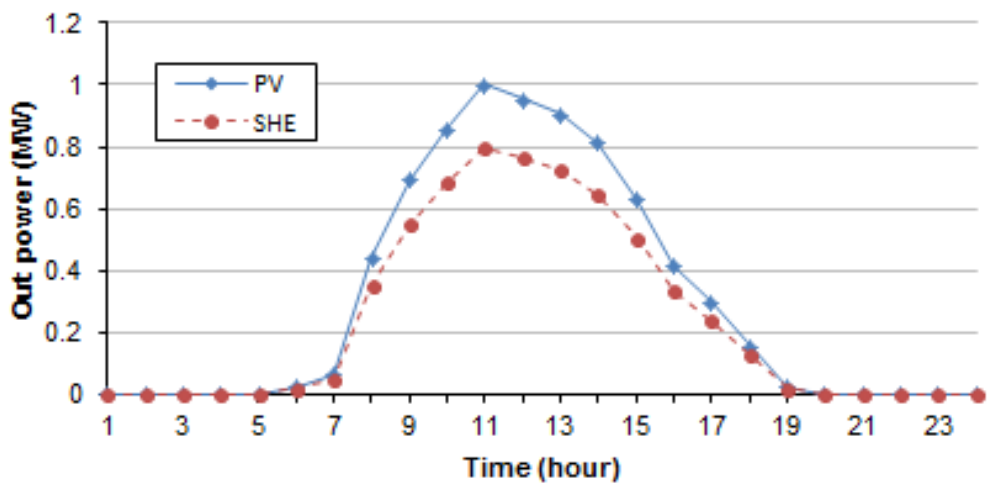

Figure 4. Output power of PV and SHE

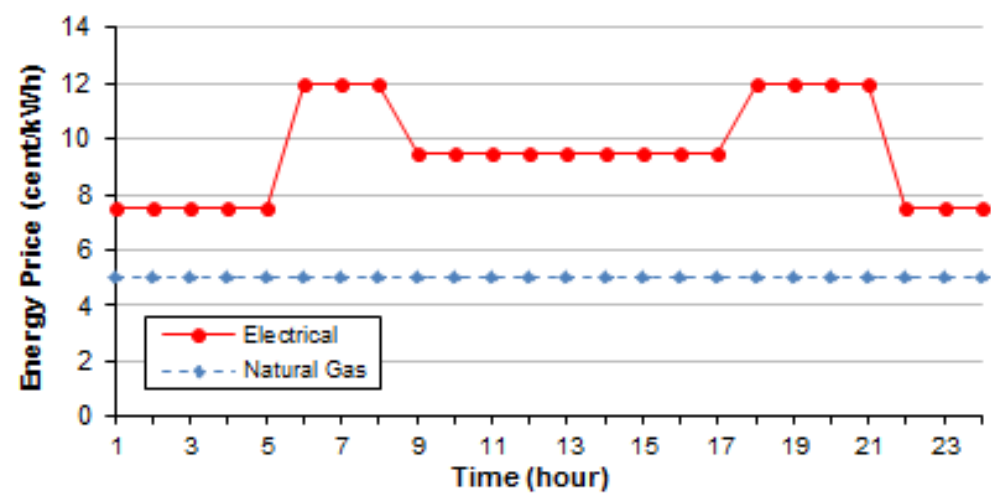

Figure 5. Electrical and natural gas price

\subsection{Sensitivity analysis on the change of electrical price}

The sensitivity of the total cost, as well as the electricity and natural gas purchased from the market of the proposed energy hub network with respect to the electrical price, is analysed. It is assumed that the electrical price varies from 0 to $150 \%$ of the base price in different analysed scenarios. The change of total cost together with the electricity and natural gas received from the supply substation of the energy hub network is expressed in figure 15.

It can be seen that at first when the price is low $(0-50 \%)$, all electricity demand should be purchased from the market and supplied by the PV. The operating power of CHP is zero and thus the all heat demand is supplied by the GB and SHE with the maximum power. The total cost of the network fast increases depending on the electrical price. When the electrical price increases $(50 \%-110 \%)$, the CHP is selected to supply both electricity and heat for loads. This means the natural gas received from the substation increases while the electricity received reduces. In this scenario, the total purchased energy increases due to the low efficiency of the CHP. However, the total cost increases more slowly due to electricity supplied from CHP with constant gas prices. When the price is from $110 \%$ and above, the CHP and GB should be operated with maximum power because the electrical price produced by CHP is cheaper than the purchase price from the market. This means the natural gas and electricity received from the substation in this scenario are constant.

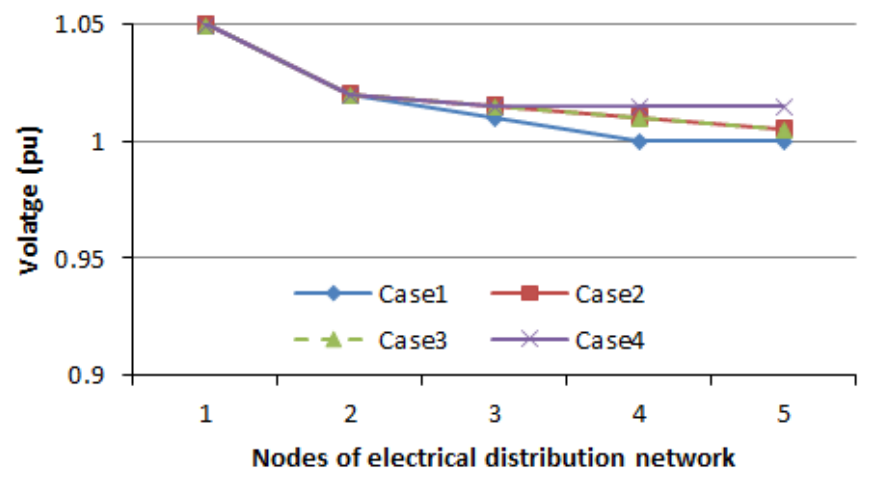

Figure 6. Comparison of node voltages 


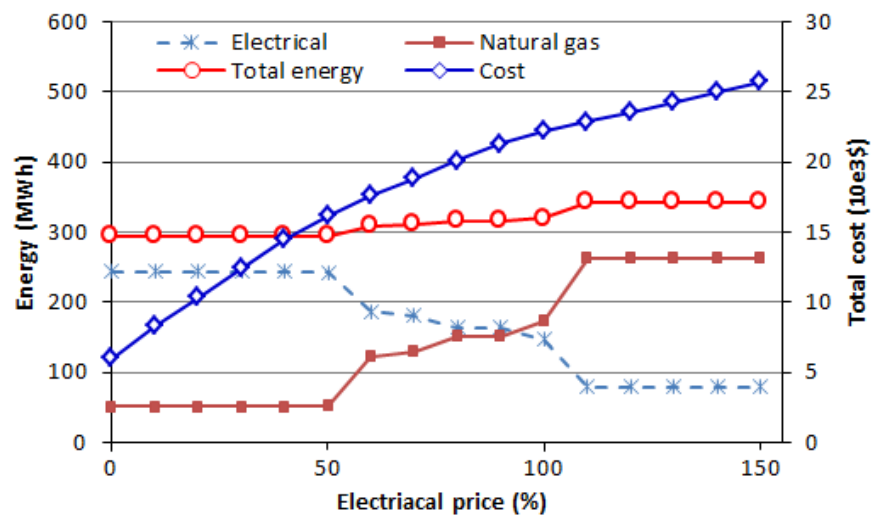

Figure 7. Change of cost and received energy of network with respect to the electrical price in case 4

\section{CONCLUSION}

The purpose of this paper is to optimize the operating schedule for the multi-energy system within the context of interaction in a network of multi-energy hubs. Hence, an optimal scheduling problem for a network of multienergy hubs considering the availability of solar and the utility energy distribution network is formulated. The problem was formulated with the objective function that minimizes the energy and operation cost of the network, the constraints of electricity, heat and cooling demand, and the constraints of the distribution network operation. The proposed model is examined in a case study of the IEEE 5-bus network structure. The numerical simulation showed significant efficiency of the conversion between energies in EHs by CHP, EC, and AC lead to reducing not only the energy cost of EHs but also the loss of distribution networks. Besides, technical parameters of both the EDN and the GDN are always guaranteed in all cases and they are improved in cases where CHP, PV, SHE, and BESS are utilized. The price sensitivity analysis also shows that CHP starts to be operated when the price is about $50 \%$ of the base price and the operation power is at the maximum level when the price is larger than $110 \%$ of the base price. Additionally, the interconnection of multi-energy hubs with conversion devices in a network still has the potential for reducing emissions. However, the uncertainty of output power of PV resource and real-time electricity price should be handled. Hence, future research work must focus on investigating the influence of different levels of variability parameters to improve the practicality of the optimal scheduling model.

\section{ACKNOWLEDGMENTS}

This work is supported by Thainguyen University of Technology (TNUT), Vietnam.

\section{REFERENCES}

[1] M. Mohammadi, Y. Noorollahi, B. Mohammadi-ivatloob, H. Yousefi, "Energy hub: From a model to a concept - A review", Renewable and Sustainable Energy Reviews, vol. 80, 2017, pp. 1512-1527.

[2] Y. Sawle, S.C. Gupta, A.K. Bohre, "Review of hybrid renewable energy systems with comparative analysis of offgrid hybrid system”, Renewable and Sustainable Energy Reviews, vol. 81(2), 2018, pp. 2217-2235.

[3] M. Mohammadi, Y.Noorollahi, B.Mohammadi-Ivatloo, "An Introduction to Smart Energy Systems and Definition of Smart Energy Hubs". In: Mohammadi-Ivatloo B., Jabari F. (eds), Operation, Planning, and Analysis of Energy Storage Systems in Smart Energy Hubs (Springer, Cham, 2018, 1-12).

[4] D.O. Gerasimov, E.V. Serdyukova, K.V Suslov, N.S. Buryanina, Y.F. Korolyuk, "Energy hub component models for multi-energy system", J. Phys.: Conf. Ser., 1582-012033, 2020.

[5] A.Maroufmashat, S.T.Taqvi, A.Miragha, M.Fowler and A.Elkamel, "Modeling and Optimization of Energy Hubs: A Comprehensive Review", Inventions, 4,50, 2019.

[6] Z. Li, W. Zhang, R. Zhang, H. Sun, "Development of renewable energy multi-energy complementary hydrogen energy system (A Case Study in China): A review”, Energy Exploration \& Exploitation, Vol. 38(6), 2020, pp. 2099 2127.

[7] C.Klemm, P.Vennemann, "Modeling and optimization of multi-energy systems in mixed-use districts: A review of existing methods and approaches", Renewable and Sustainable Energy Reviews 135, 110206, 2021.

[8] T. Wakui, R. Yokoyama, "Optimal structural design of residential cogeneration systems in consideration of their operating restrictions", Energy, vol. 64, 2014, pp. 719-733.

[9] T.T. Ha, Y. Zhang, V.V. Thang, J. Huang, "Energy hub modeling to minimize residential energy costs considering solar energy and BESS", J. Mod. Power Syst. Clean Energy, vol. 5(3), 2017, pp. 389-399.

IJEEI, Vol. 9, No. 2, June 2021: 276-288 
[10] M.S. Javadi, A.A. Moghaddam, G.M. Guerrero, "Optimal scheduling of a multi-carrier energy hub supplemented by battery energy storage systems", IEEE International Conference on Environment and Electrical Engineering, 69 June 2017, Italy.

[11] M. Dolatabadi, A. Dolatabadi, "Optimal electrical and thermal energy management of a residential energy hub in the presence of PV systems", Journal of Environmental Science, 2018, pp. 1-4.

[12] A.Eshraghi, G.Salehi, S.Heibati, K.Lari, "An enhanced operation model for energy storage system of a typical combined cool, heat and power based on demand response program: The application of mixed integer linear programming”, Building Services Engineering Research and Technology, Vol. 40(1), 2019, pp, 47-74.

[13] F. Jamalzadeha, A.H. Mirzahosseini, F. Faghihi, M. Panahi, "Optimal operation of energy hub system using hybrid stochastic-interval optimization approach", Sustainable Cities and Society, vol. 54, 2020, pp. 1-9.

[14] M.J. Vahid-Pakdel, S. Nojavan, B. Mohammadi-ivatloo, K. Zare, "Stochastic optimization of energy hub operation with consideration of thermal energy market and demand response", Energy Conversion and Management, vol. 145, 2017, pp. 117-128.

[15] A.Najafi-Ghaleloua, S. Nojavan, K. Zarea, B. Mohammadi-Ivatlooa, "Robust scheduling of thermal, cooling and electrical hub energy system under market price uncertainty", Applied Thermal Engineering, 149, 2019, pp. 862880.

[16] M.S. Javadi, M. Lotfi, A.E.Nezhad, A. Anvari-Moghaddam, J.M. Guerrero, "Optimal Operation of Energy Hubs Considering Uncertainties and Different Time Resolutions”, IEEE Transactions on Industry Applications, Vol. 56, 5, 2020, pp. $5543-5552$.

[17] D. Rakipourab, H. Baratia, "Probabilistic optimization in operation of energy hub with participation of renewable energy resources and demand response", Energy, Vol. 173, 15, 2019, pp. 384-399.

[18] Z.Yuana, S.Hea, A. Alizadeh, S. Nojavand, K. Jermsittiparsert, "Probabilistic scheduling of power-to-gas storage system in renewable energy hub integrated with demand response program”, Journal of Energy Storage 29, 101393, 2020, pp. 1-15.

[19] M. Moeini-Aghtaie, A. Abbaspour, M. Fotuhi-Firuzabad, E. Hajipour," A Decomposed Solution to Multiple-Energy Carriers Optimal Power Flow", IEEE Transactions on Power Systems, vol. 29(2), 2014, pp. 707-716.

[20] S.A Kalogirou, "Solar Energy Collectors". In: Kalogirou SA, Solar Energy Engineering, 2015, pp. 125-220.

[21] Y. Jiang, F. Mei, J. Lu and J. Lu, "Two-Stage Joint Optimal Scheduling of a Distribution Network With Integrated Energy Systems", IEEE Access, vol. 9, 2021, pp. 12555-12566.

[22] O. Dzobo and X. Xia, "Optimal operation of smart multi-energy hub systems incorporating energy hub coordination and demand response strategy", Journal of Renewable and Sustainable Energy 9 (4), 2017, pp.1-27.

[23] Y. Huang, W. Zhang, , K. Yang, W. Hou, Y. Huang, "An Optimal Scheduling Method for Multi-Energy Hub Systems Using Game Theory”, Energies, vol. 12(12), 2019, pp. 2270.

[24] A. Maroufmashat, M. Fowler, K.S. Sattari, A. Elkamel, R. Roshandel, A. Hajimiragha, "Mixed integer linear programing based approach for optimal planning and operation of a smart urban energy network to support the hydrogen economy", International Journal of Hydrogen Energy, vol. 41(19), 2016, pp. 7700-7716.

[25] A. Maroufmashat, A. Elkamel, A. Fowler, A. Sattari, R. Roshandel, A. Hajimiragha, A. Walker, E. Entchev, "Modeling and optimization of a network of energy hubs to improve economic and emission considerations", Energy, vol. 93, 2015, pp. 2546-2558.

[26] A.A. Eladl, M.I. El-Afifib, M.A. Saeeda, M. M. El-Saadawi, "Optimal operation of energy hubs integrated with renewable energy sources and storage devices considering CO2 emissions", Electrical Power and Energy Systems 117(105719), 2020, pp. 1-17

[27] P. Mancarella, "MES (multi-energy systems): An overview of concepts and evaluation models", Energy, 2014, vol. 65, pp. 1-17.

[28] W. Huang, N. Zhang, J. Yang, Y. Wang, C. Kang, "Optimal Configuration Planning of Multi-Energy Systems Considering Distributed Renewable Energy”, IEEE Transactions on Smart Grid, vol. 10(2), 2017, pp. 1452-1464.

[29] Y. Guo, C. Zhao, "Islanding-Aware Robust Energy Management for Microgrids", IEEE Transactions on Smart Grid, vol. 9(2), 2018, pp. 1301-1309.

[30] J.L.R. Duarte, N. Fan, "Operations of a microgrid with renewable energy integration and line switching", Energy System, vol. 10, 2019, pp. 247-272.

[31] A.L. Bukar, C.W. Tan, K.Y. Lau, "Optimal sizing of an autonomous photovoltaic / wind / battery / diesel generator microgrid using grasshopper optimization algorithm", Solar Energy, vol. 188, 2019, pp. 685-696.

[32] D. Wanga, J. Qiub, L. Reedmana, K. Meng, L.L. Lai, "Two-stage energy management for networked microgrids with high renewable penetration”, Applied Energy, vol. 226, 2018, pp. 39-48.

[33] C.R. Martinez-Mares, "Fuerte-Esquivel, A unified gas and power flow analysis in natural gas and electricity coupled networks", IEEE Trans. Power Syst., vol. 27(4), 2012, pp. 2156-2166.

[34] S. Deng, L.L. Wu, F. Wei, Q.H. Wu, Z.X. Jing, X.X. Zhou, M.S. Li, "Optimal Operation of Energy Hubs in an Integrated Energy Network Considering Multiple Energy Carriers”, 2016 IEEE Innovative Smart Grid Technologies - Asia (ISGT-Asia), Nov 28 - Dec 1, 2016, Melbourne, Australia.

[35] J.Kronqvist, D.E. Bernal, A. Lundell, I.E. Grossmann, "A review and comparison of solvers for convex MINLP", Optimization and Engineering, vol.20, 2019, pp. 397-455.

[36] M.Y.Selim, A. Alsharoa, A.E. Kamal, M.A. Alnuem, "SURE: A Novel Approach for Self Healing Battery Starved Users Using Energy Harvesting", IEEE Access, vol.5, 2017, pp.6110-6120.

[37] Gams.com [Internet]. "General algebraic modeling system". [Retrieved January 2020] Available from: http://www.gams.com/dd/docs/bigdocs/GAMSUsersGuide.pdf . 
[38] S.Sutradhar, N.B. Dev Choudhury, N. Sinha, "MINLP for hydrothermal scheduling problem considering transmission loss", 2016 IEEE Annual India Conference (INDICON), 16-18 Dec. 2016, India.

[39] P.K. Gouda, A.K. Sahoo, P.K. Hota, "Optimal Power Flow Including Unified Power Flow Controller in a Deregulated Environment”, International Journal of Applied Engineering Research, vol. 10(1), 2015, pp. 505-522.

[40] Z.A. Ashokrao, P.R. Machhindra, S. Padmanaban, M.S. Bhaskar, L. Martirano, Z. Leonowicz, "Realization of 5-bus System Using Soft Computing Technique for Flexible Alternating Current Transmission System (FACTS) Devices", 2017 IEEE International Conference on Environment and Electrical Engineering, 6-9 June 2017, Italy.

[41] S. Hasanpour, R. Ghazi, M.H. Javidi, "A New Approach for Accurate Pricing of Reactive Power and Its Application to Cost Allocation in Deregulated Electricity Markets", Journal of Iranian Association of Electrical and Electronics Engineers, vol. 7(2), 2010, pp. 31-39.

[42] R. Kumar, A. Kumar, "Real and reactive power price of generators to load in pool based electricity market model", 2015 International Conference on Energy Economics and Environment (ICEEE), 27-28 March 2015, India.

[43] S. Deng, Q. Wu, Z. Jing, L. Wu, F. Wei, X. Zhou, "Optimal Capacity Configuration for Energy Hubs Considering Part-Load Characteristics of Generation Units", Energies, vol. 10(1966), 2017, pp. 1-29.

[44] K. Amiri, T. Niknam, "Optimal Planning of a Multi-carrier Energy Hub Using the Modified Bird Mating Optimizer, Iranian Journal of Science and Technology", Transactions of Electrical Engineering, vol. 43 (1), 2019, pp. 517-526.

[45] A.A. Bayod-Rújula, Y. Yuan, A. Martínez-Gracia, J. Wang, J. Uche, H. Chen, "Modelling and Simulation of a Building Energy Hub", Proceedings, vol. 2(1431), 2018, pp. 1-6.

\section{BIOGRAPHY OF AUTHORS}

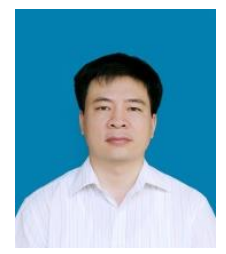

V. V. Thang received the B.Sc. and M.Sc. degrees in the Department of Electric Power Systems, Thai Nguyen University of Technology (TNUT), Vietnam, in 2001 and 2007, respectively. He received the Ph.D. Degree at the Hanoi University of Science and Technology (HUST) in 2015. He is currently a lecturer in the Department of Electric Power System at TNUT. His major research interests include optimizing energy systems, distributed generations and distribution systems in deregulated electricity markets. 